\title{
SPAN OF LENS SPACES
}

\section{KOICHI IWATA}

ABstract. We prove that the span (i.e., maximal number of linearly independent vector fields) of $(2 n+1)$-dimensional lens spaces $L^{n}(p)$, where $p$ is an odd prime and where $n+1=m \cdot 2^{t}$ ( $m$ odd), is equal to the span of $(2 n+1)$-dimensional sphere if $t+1 \equiv 0,1,2(\bmod 4)$ or if $n>3, t+1 \equiv 3(\bmod 4)$, and $p \geqq t+3$. This result is an improvement of a theorem given by $\mathrm{T}$. Yoshida.

Let $L^{n}(p), p$ an odd prime, be a $(2 n+1)$-dimensional (standard) lens space. We define span of a closed connected manifold $M$ to be the maximal number of linearly independent vector fields on $M$.

In this note, using an obstruction theory adopted by Denis Sjerve [1], we prove the following:

THEOREM. Let $n+1=m \cdot 2^{t}(m$ odd $)$. If $t+1 \equiv 0,1$ or $2, \bmod 4$, then $\operatorname{span}\left(L^{n}(p)\right)=\operatorname{span}\left(S^{2 n+1}\right)$. If $t+1 \equiv 3, \bmod 4$, and $n>3$, then $\operatorname{span}\left(S^{2 n+1}\right)-1 \leqq \operatorname{span}\left(L^{n}(p)\right) \leqq \operatorname{span}\left(S^{2 n+1}\right)$. Moreover for $p \geqq t+3$, we have $\operatorname{span}\left(L^{n}(p)\right)=\operatorname{span}\left(S^{2 n+1}\right)$.

This is an improvement of a theorem of T. Yoshida [2], which asserts that

(i) $\operatorname{span}\left(S^{2 n+1}\right)-1 \leqq \operatorname{span}\left(L^{n}(p)\right) \leqq \operatorname{span}\left(S^{2 n+1}\right)$, if $t+1 \equiv 0(\bmod 4)$.

(ii) $\operatorname{span}\left(L^{n}(p)\right)=\operatorname{span}\left(S^{2 n+1}\right)$, if $t+1 \equiv 1$, or $2(\bmod 4)$.

(iii) $\operatorname{span}\left(S^{2 n+1}\right)-2 \leqq \operatorname{span}\left(L^{n}(p)\right) \leqq \operatorname{span}\left(S^{2 n+1}\right)$, if $t+1 \equiv 3(\bmod 4)$.

Especially, $\operatorname{span}\left(L^{3}(3)\right)=5=\operatorname{span}\left(S^{7}\right)-2$.

Proof of THE Theorem. By Yoshida's theorem, we have to prove our theorem only for the case $t+1 \equiv 0$ or $3, \bmod 4$.

First, assume $t+1 \equiv 0, \bmod 4$. Then $\operatorname{span}\left(S^{2 n+1}\right)=2 t+2$. Let $\xi$ be $V_{2 n+1,2 t+2}$-bundle associated with principal $S O(2 n+1)$-bundle over $L^{n}(p)$. It is sufficient to prove that $\xi: L^{n}(p) \rightarrow B S O(2 n+1)$ can be lifted to $L^{n}(p) \rightarrow B S O(2 n+1-2 t-2)$. By [1, Lemma (2.2)], $\pi_{i}\left(V_{2 n+1,2 t+2}\right)$ has no $p$-part for $i \leqq 2 n$ and is finite for $i<4(n-t)-1$. Since $t \geqq 3, t+1 \equiv 0, \bmod 4$, we have $4(n-t)-1>2 n$. Therefore, just as in the proof of Theorem A in [1], we find that the obstructions to the above lifting vanish.

Next we assume $t+1 \equiv 3, \bmod 4$. In this case $\operatorname{span}\left(S^{2 n+1}\right)=2 t+3$. If $n>3$, we have $\operatorname{span}\left(L^{n}(p)\right) \geqq 2 t+2$, just as above. Now let $\xi$ be

Received by the editors September 4, 1969.

AMS 1969 subject classifications. Primary 5730.

Key words and phrases. Lens space, linearly independent vector field, associated principal bundle. 
$V_{2 n+1,2 t+3}$-bundle associated with principal $S O(2 n+1)$-bundle over $L^{n}(p)$. Consider the obstruction to lifting $\xi: L^{n}(p) \rightarrow B S O(2 n+1)$ to $L^{n}(p) \rightarrow B S O(2 n+1-2 t-3)$. If $p \geqq t+3$, then $\pi_{i}=\pi_{i}\left(V_{2 n+1,2 t+3}\right), i \leqq 2 n$, has no $p$-part and is finite with the exception of $\pi_{2(n-t)-2}=Z[1$, Lemma (2.2)]. Therefore, in the diagram

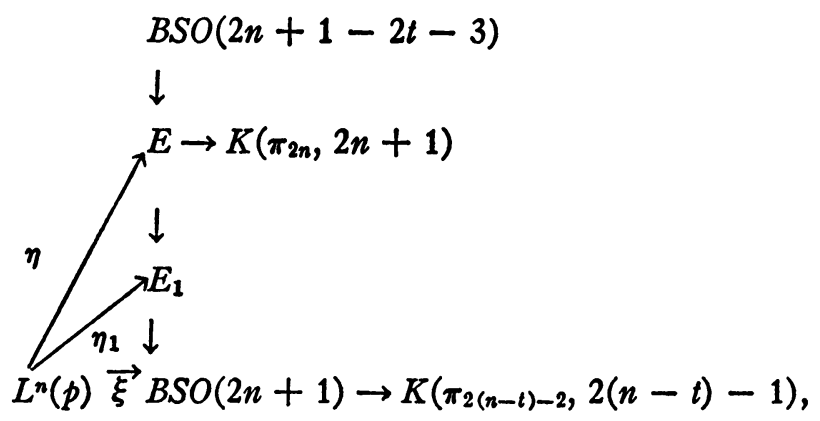

$\xi$ can be lifted to $\eta_{1}$, since $H^{2(n-t)-1}\left(L^{n}(p), Z\right)=0$. The fact that $\eta_{1}$ is liftable to $\eta$ and $\eta$ is liftable to $L^{n}(p) \rightarrow B S O(2 n+1-2 t-3)$ is easily proved as in the former case.

\section{REFERENCES}

1. Denis Sjerve, Geometric dimension of vector bundles over lens spaces, Trans. Amer. Math. Soc. 134 (1968), 545-557. MR 38 \#1695.

2. Toshio Yoshida, A remark on vector fields on lens spaces, J. Sci. Hiroshima Univ. Ser. A-I Math. 31 (1967), 13-15. MR 35 \#7349.

College of General Education, Tohoku University, Sendai, Japan 